\title{
BMJ Open Health outcomes of unpaid care workers in low-income and middle-income countries: a protocol for a systematic review
}

Irene Magaña, ${ }^{1,2}$ Pablo Martínez, ${ }^{1,3,4}$ María-Soledad Loyola ${ }^{1,2}$

To cite: Magaña I, Martínez $P$, Loyola M-S. Health outcomes of unpaid care workers in lowincome and middle-income countries: a protocol for a systematic review. BMJ Open 2018:8:e018643. doi:10.1136/ bmjopen-2017-018643

- Prepublication history and additional material for this paper are available online. To view these files, please visit the journal online (http://dx.doi. org/10.1136/bmjopen-2017018643).

Received 16 August 2017 Revised 13 December 2017 Accepted 15 December 2017

Check for updates

${ }^{1}$ Escuela de Psicología, Facultad de Humanidades, Universidad de Santiago de Chile, Santiago Chile

${ }^{2}$ Centro de Estudios Migratorios (CEM), Universidad de Santiago de Chile, Santiago, Chile

${ }^{3}$ Centro de Innovación en Tecnologías de la Información para Aplicaciones Sociales CITIAPS, Universidad de Santiago de Chile, Santiago, Chile

${ }^{4}$ Instituto Milenio para la Investigación en Depresión y Personalidad (MIDAP), Santiago, Chile

Correspondence to

Dr Irene Magaña;

irene.magana@usach.cl

\section{ABSTRACT}

Introduction The literature on health outcomes of unpaid care work has included studies coming from high-income countries, and has reported gender inequalities that make caregiving women more vulnerable to physical and mental health problems. The impact of unpaid care work on the health of those living in low-income and middle-income countries, where women's autonomy is more limited, is unknown.

Methods and analysis We will conduct a systematic review of observational studies on health outcomes according to unpaid caregiving status and sex of people living in low-income and middle-income countries. Cumulative Index to Nursing and Allied Health Literature, PubMed and Scientific Electronic Library Online Citation Index will be searched for reports in English or Spanish with published results from inception to 1 June 2017. We expect the studies to have recruited individuals in lowincome and middle-income countries, including exposed and non-exposed groups to participation in unpaid care to members if their households or community reporting either physical and/or mental health problems, self-reported health-related quality of life, self-care skills/behaviours or use of any healthcare services in the participants. Data extraction, the assessment of risk of bias and confounding, and qualitative synthesis will be carried out by two independent reviewers with the assistance of a third party. Dissemination Results are expected to be published in peer-reviewed journals from the field of health and gender, or health and inequality.

PROSPERO registration number CRD42017071785.

\section{INTRODUCTION}

The inequality of opportunities for participating in decision-making processes, exercise of power and access to health resources differentially exposes people to health risks. ${ }^{2}$ This social stratification occurs throughout categories such as social class, gender, ethnicity and sexuality, among others, introducing undesirable inequalities in health, constituting a violation of the right to health. ${ }^{12}$ Acting on these social determinants of health involves redistributing power for the benefit of the most vulnerable groups. ${ }^{2}$

\section{Strengths and limitations of this study}

- The Preferred Reporting Items for Systematic Review and Meta-Analysis Protocols checklist and recommendations from the Meta-analysis of Observational Studies in Epidemiology group were used to strengthen the publication of this protocol.

- There have been no recent syntheses of the evidence on the state of health of unpaid caregivers in lowincome and middle-income countries, nor on the gender differences in the health status of caregivers who come from these countries.

- Recommendations from the Cochrane Collaboration and the Agency for Healthcare Research and Quality were used to design this systematic review.

- This systematic review will be limited to published articles; no grey literature will be searched.

- Searches will be limited to studies published in English and Spanish languages.

Unequal social relations between sexes shape the status of political, economic and social subordination of women, ${ }^{3}$ affecting their autonomy - which is made up of capabilities and conditions to freely make decisions that have an impact on their lives ${ }^{4}$ - and that cause disparities in health results, worse economic and social consequences of poor health, and health systems with ineffective resolving power. ${ }^{5}$ As one of the most influential social determinants of health, attaining gender equity has been recognised as a development goal. ${ }^{6}$

Unpaid care work, defined as the service of health maintenance, well-being and the protection of family and community members, ${ }^{7}$ is an activity that is determined by gender, with more women devoting time to it than men. ${ }^{8}$ In spite of the huge contribution to unpaid care work to producing health and sustainable development of communities, the lack of recognition, valuation and support to those who are devoted to these tasks, has 
made women more vulnerable to physical and mental health problems. ${ }^{5}$

Additionally, systematic reviews and meta-analyses have confirmed that the unpaid care is related to higher levels of stress hormones and worse response of antibodies, greater prevalence of behavioural risk factors for chronic diseases, such as unhealthy diets and sedentary lifestyles and greater use of health services compared with subjects that do not provide these services. ${ }^{9}{ }^{10}$ Moreover, a longitudinal study showed that unpaid caregivers end up being poorer and less happy and healthy than their non-caregiving counterparts. ${ }^{11}$

Unpaid care work is costly. According to the Global Valuing the Invaluable Analysis, which measured the economic contribution of paid and unpaid work that women make to the health sector, unpaid care by women, after accounting for gender wage differentials and social security benefits, could be valued at 3.09\% of the global gross domestic product, ${ }^{12}$ invisibly subsidising the health sector because it is considered in national accounts. ${ }^{12}$

Women's autonomy is more limited in low-income and middle-income countries (LMICs), ${ }^{13}$ devoting more hours to unpaid work than women in high-income countries (HICs). This is due to the lower participation of men in LMICs in these tasks. ${ }^{812}$ Additionally, it has been reported that the objective and subjective burden due to unpaid care work is greater in LMICs than in HICs, representing a decline for family economy, and being greater for female caregiver than for male caregivers. ${ }^{14}$

In spite of this scenario, literature syntheses on health outcomes of unpaid care work have included participants who come from HICs, ${ }^{9}{ }^{10}$ and have made comparisons between the sexes, confirming the worst outcomes for women. The only review carried out in developing countries focused on recipients of care for the chronically ill or disabled, and did not focus on the comparison between unpaid care workers and those who do not participate in these tasks. ${ }^{15}$

Therefore, the specific contribution of unpaid care work in health in LMICs cannot be elucidated by the current status of the synthesis of evidence. Furthermore, considering the differences in health as a result of the sex of the caregiver may facilitate the comparison with the HICs and help define future intervention strategies in countries in which the limited autonomy of women could considerably affect their state of health.

\section{Objectives}

The objective of this systematic review is to evaluate the health outcomes associated with unpaid care work in LMICs, taking into account sex differences. This systematic review will aim to answer the following question:

1. What are the health outcomes of unpaid care workers compared with those who do not perform unpaid care activities in LMICs?

2. Do health outcomes of unpaid care workers from LMICs differ by sex?

\section{METHODS AND ANALYSIS}

The protocol of this systematic review followed the guidelines of the Preferred Reporting Items for Systematic Review and Meta-Analysis Protocols checklist ${ }^{16}$ and some of the recommendation of the Meta-analysis of Observational Studies in Epidemiology group, which were adapted. ${ }^{17}$

\section{Study eligibility criteria}

Participants

Individuals without distinction of age, sex or ethnicity, living in LMICs.

\section{Exposure}

Provision of unpaid care to household or community members. For the purposes of this systematic review, unpaid care work was defined as the set of activities carried out for the development and well-being of the everyday life of members of the home or the community. Unpaid care work may involve the care of people who are: (1) affected by permanent health problems or condition of dependency; (2) children aged between 0 and 14; (3) healthy people aged between 15 and 65, who do not require special care or have symptoms clinically identifiable diseases; and (4) adults over 65 years of age. This conceptualisation has been used in time-use surveys in Chile. ${ }^{18}$

\section{Comparators}

Individuals who do not provide unpaid care to household or community members. This may include subjects that do not qualify as unpaid caregivers according to a certain threshold of hours of unpaid care work provided, according to the study definition. Also, in the case of the longitudinal studies without a control group, it can refer to the subjects who acted as their own control, based on the change of the status of unpaid care provider.

\section{Outcomes}

The studies must include at least one of the following outcomes: physical and/or mental health problems, health-related quality of life, self-care behaviour or skills and use of health services. These outcomes must use objective or self-reported measures.

\section{Study design}

Observational studies (eg, case series, cross-sectional study, case control study and cohort studies). Additionally, systematic reviews and meta-analyses of observational studies will be included. Controlled trials will be excluded.

\section{Context}

General population or clinical samples LMICs, according to the World Bank income classification. ${ }^{19}$

\section{Report eligibility criteria}

Completed studies with published results, in English or Spanish, from their inception to 1 June 2017, will be included. Study protocols will be excluded. 


\section{Information sources}

The Cumulative Index to Nursing and Allied Health Literature, PubMed, and Scientific Electronic Library Online Citation Index will be searched. Additionally, reference lists of included studies will be screened for relevant papers.

\section{Search strategy}

The search strategy is presented in the online supplementary file. Terms related to exposure (eg, "caregivers" and "unpaid"), outcomes (eg, "State of health" and "self-care") and contexts (eg, "low and middle income countries") will be used. In addition, a series of terms will be used to exclude reports of studies according to their design (ie, studies that assessed the effectiveness of interventions, or qualitative studies).

\section{Study records}

All study records will be imported into EndNote Web and duplicates will be removed. The study selection process will be carried out in two stages (screening of title/ abstract, and full-text assessment of articles), with two reviewers independently, and in duplicate, determining inclusion/exclusion of study records based on previously specified criteria. If discrepancies arise during any stage, these will be solved by discussion and confirmed by a third reviewer. Finally, data from multiple records of the same study will be pieced together.

Data extraction will be carried out using a standardised sheet recommended by the Cochrane Handbook of Systematic Reviews of Interventions, ${ }^{20}$ following the same reviewing process described above (two independent reviewers and a third to resolve any disagreements), with regular meetings held to verify the quality of the extracted data. The following data will be extracted from each study included in this review: (1) first author, year of publication and country of origin; (2) participants inclusion/exclusion criteria (age, sex, ethnicity, socioeconomic status and/or urban/rural residence) and setting (eg, general population or clinical sample); (3) exposure/comparison characteristics, including criteria for classification as unpaid care worker (eg, number of hours) and type of care tasks provided; (4) type of outcomes reported (ie, clinical, patient-reported or use of healthcare services), instruments, follow-up periods (in case of longitudinal studies) and main findings (with effect size, CIs, and statistical significance) and (5) type of study design.

\section{Outcomes and prioritisation}

Included studies must report any outcome of interest, and, in the case of longitudinal studies, baseline levels of these outcomes must be reported. Due to the inclusion of cross-sectional and longitudinal studies, no distinction based on follow-up periods will be made. The primary outcome will be mental and/or physical health problems of participants measured either objectively or by self-report of symptoms or illnesses. Secondary outcomes will be: (1) self-reported health-related quality of life; (2) self-care skills/behaviours and (3) use of any healthcare services.

\section{Risk of bias: individual studies}

To assess the risk of bias and confounding in the included studies, we will use the 'Item Bank for Assessing Risk of Bias and Confounding for Observational Studies of Interventions or Exposure' developed by the Agency for Healthcare Research and Quality. ${ }^{21}$

This tool includes an assessment of 13 sources of bias and confounding: variations across groups of the study in the inclusion/exclusion criteria, the recruitment strategies or the length of follow-up, inappropriate selection of the comparison group, failing to account for important variations in the execution of the study, outcome assessor not blinded to exposure status of participants, study measures of dubious validity and reliability or implemented inconsistently, impact of high or differential loss to follow-up not assessed, important outcomes or harms/adverse events not reported, study limitations hampers the credibility of the study, no documented attempts to balance the allocation between the groups and important confounding variables not taken into account. ${ }^{21}$

The same procedure described above for the study selection and the data extraction will be employed to assess the risk of bias of the included studies, with two independent reviewers and a third to resolve any disagreements.

\section{Data synthesis}

A qualitative synthesis of the included studies will be conducted to provide an overview of the differences in health outcomes among participants coming from LMICs, taking into account the study characteristics and risk of bias/confounding. Sex differences in health outcomes among unpaid caregivers living in these countries will also be explored. These results will be summarised using comparative tables recommended in the Cochrane Handbook for Systematic Reviews of Interventions. ${ }^{20}$

\section{DISCUSSION}

For over two decades, the United Nations have called on member states to include unpaid work in economic and vital statistics. ${ }^{22} 23$ Today, in the Region of Latin America, a small number of countries have national time-use surveys, and very few have implemented a nationwide system of care. This scenario is replicated in other regions of the developing world. ${ }^{24}$ The protocol of a systematic review reported here echoes this reality and aims to contribute to the acknowledgement and valuation of unpaid care work in LMICs.

It is expected that the political and economic obstacles to overcome the unequal division of unpaid work, particularly in LMICs, will be expressed in a small number of studies that compare caregivers and non-caregivers, 
being these publications rather recent. Additionally, the socioeconomic and geographical variety among the LMICs will help to understand how the exercise of care is related to other sociocultural variables.

The authors of this systematic review anticipate limitations due to the eligibility criteria and the information sources used. By limiting publications to the English or Spanish languages, and the scope of studies published in other languages (eg, Chinese or Arabic), a geographical bias in favour of evidence from Latin America may have been introduced. This decision has been made considering the financial resources and the time set for this systematic review.

Again, for reasons of economy of resources for this systematic review, we have opted for the design of a search strategy that excludes terms related to experimental studies. In this regard, Cochrane Collaboration recognises that the process of identifying non-random studies in searches and their subsequent selection may be prone to biases due to lack of a predefined search strategy and to report highly heterogeneous characteristics of the study design, compared with the randomised clinical trials. $^{20}$

Finally, this systematic review was limited to the review of the evidence published in the databases mentioned in the 'Information Sources' section. This will hinder the detection of selection biases because grey literature search will not be conducted, nor will the authors and authorities be contacted on the subject to identify additional sources, due to the previously mentioned reasons.

\section{ETHICAL CONSIDERATIONS}

As this study is a systematic review, there is no requirement for ethical review and approval.

\section{DISSEMINATION}

The results of this systematic review will be published in peer-reviewed journals covering topics such as: gender and health, and health inequalities.

\section{AMENDMENTS}

This section will include any changes introduced to the protocol after its publication. A set of amendments to the protocol that were carried out are listed below:

- The lower age limit (18 years and over) was eliminated to include all participants regardless of age.

- The reference to the fact that participants may or may not carry out unpaid care work was eliminated. These conditions apply and were duly developed in the description of the exposure and comparator.

Contributors IM is the guarantor of this systematic review and provides her expert opinion during the reviewing process; PM and M-SL gave valuable input to the design of this systematic review, and will be in charge of the data extraction and risk of bias assessment under the supervision of IM. All authors will contribute equally to data synthesis. All authors edited, modified and approved the final version of this manuscript.

Funding This systematic review is financed by the Office of Scientific and Technological Research (DICYT) of the Vice-Rector of Research, Development and Innovation at the University of Santiago of Chile, through Projects 021793MF_CONT and 031693MF.

Disclaimer The funding institution is not involved in any part of this study. Competing interests None declared.

Patient consent Not required.

Provenance and peer review Not commissioned; externally peer reviewed.

Open Access This is an Open Access article distributed in accordance with the Creative Commons Attribution Non Commercial (CC BY-NC 4.0) license, which permits others to distribute, remix, adapt, build upon this work non-commercially, and license their derivative works on different terms, provided the original work is properly cited and the use is non-commercial. See: http://creativecommons.org/ licenses/by-nc/4.0/

(c) Article author(s) (or their employer(s) unless otherwise stated in the text of the article) 2018. All rights reserved. No commercial use is permitted unless otherwise expressly granted.

\section{REFERENCES}

1. Marmot M, Wilkinson R. Social determinants of health. $2^{\text {nd }}$ ed. Oxford: Oxford University Press, 2006.

2. Solar O, Irwin A. A conceptual framework for action on the social determinants of health. Social determinants of health discussion paper 2 (policy and practice). Geneva: World Health Organization, 2010.

3. Pan American Health Organization [PAHO]. Guide for analysis and monitoring of gender equity in health policies. Washington, DC: PAHO, 2008.

4. Economic Commission for Latin America and the Caribbean. Equality and women's autonomy in the sustainable development agenda. Santiago: United Nations, 2016.

5. Sen G, Östlin P, Unequal GA. unfair, ineffective and inefficient. Gender inequality in health: why it exists and how we can change it. Final report to the WHO Commission on Social Determinants of Health. Sweden: WGEKN, 2007.

6. United Nations General Assembly. Transforming our World: The 2030 Agenda for Sustainable Development. A/70/L.1. New York: United Nations, 2015.

7. Elson D. Progress of the world's women 2000. UNIFEM biennial report. New York: United Nations Development Fund for Women, 2000.

8. Ferrant G, Pesando LM, Nowacka K. Unpaid care work: the missing link in the analysis of gender gaps in labour outcomes: OECD Development Centre, 2014

9. Pinquart M, Sörensen S. Differences between caregivers and noncaregivers in psychological health and physical health: a metaanalysis. Psychol Aging 2003;18:250-67.

10. Vitaliano PP, Zhang J, Scanlan JM. Is caregiving hazardous to one's physical health? A meta-analysis. Psychol Bull 2003;129:946-72.

11. Carmichael F, Ercolani MG. Unpaid caregiving and paid work over life-courses: Different pathways, diverging outcomes. Soc Sci Med 2016;156:1-11.

12. Langer A, Meleis A, Knaul FM, et al. Women and Health: the key for sustainable development. Lancet 2015;386:1165-210.

13. Osamor PE, Grady C. Women's autonomy in health care decisionmaking in developing countries: a synthesis of the literature. Int J Womens Health 2016;8:191-202.

14. Shahly V, Chatterji S, Gruber MJ, et al. Cross-national differences in the prevalence and correlates of burden among older family caregivers in the World Health Organization World Mental Health (WMH) Surveys. Psychol Med 2013;43:865-79.

15. Thrush A, Hyder AA, Hyder A. The neglected burden of caregiving in low- and middle-income countries. Disabil Health $J$ 2014;7:262-72.

16. Shamseer L, Moher D, Clarke M, et al. Preferred reporting items for systematic review and meta-analysis protocols (PRISMA-P) 2015: elaboration and explanation. BMJ 2015;349:g7647.

17. Stroup DF, Berlin JA, Morton SC, et al. Meta-analysis of observational studies in epidemiology: a proposal for reporting. Meta-analysis of Observational Studies in Epidemiology (MOOSE) group. JAMA 2000;283:2008-12. 
18. Instituto Nacional de Estadísticas de Chile. Documentos de principales resultados ENUT 2015. Santiago Chile: Departamento de Estudios Sociales, Instituto Nacional de Estadísticas, 2016.

19. United Nations. World economic situation and prospects 2017. New York: United Nations, 2017.

20. Higgin JPT, Green S, eds. Cochrane handbook for systematic reviews of interventions version 5.1.0. The cochrane collaboration. 2011. www. cochrane-handbook.org. (updated Mar 2011).

21. Viswanathan M, Berkman ND, Dryden DM, et al. Assessing risk of bias and confounding in observational studies of interventions or exposures: further development of the RTI item Bank. Rockville MD: Agency for Healthcare Research and Quality (US), 2013.

22. United Nations. Report of the International Conference on Population and Development. Cairo, 5-13 September 1994. New York: United Nations, 1995

23. United Nations. Report of the Fourth World Conference on Women. Bejing, New York: United Nations, 1996. 4-15 September 1995

24. Batthyány K. Las políticas y el cuidado en América Latina: una mirada a las experiencias regionales. Asuntos de Género 2015;124. 